\title{
Hydrothermal synthesis and solid-state laser refrigeration of ytterbium-doped potassium lutetium fluoride (KLF) microcrystals
}

Xiaojing Xia, ${ }^{\dagger}$ Anupum Pant, ${ }^{\ddagger}$ Xuezhe Zhou, ${ }^{\ddagger}$ Elena A. Dobretsova,$\stackrel{\ddagger}{\ddagger}$ Alexander

B. Bard, "Matthew B. Lim,, Joo Yeon D. Roh, "Daniel R. Gamelin, " and Peter J. Pauzauskie ${ }^{*, \$, \S}$

$\dagger$ Molecular Engineering 83 Science Institute, University of Washington, Seattle, WA 98195, USA

$\ddagger$ Department of Materials Science \& Engineering, University of Washington, Seattle, WA 98195, USA

IDepartment of Chemistry, University of Washington, Seattle, WA 98195, USA

$\S$ Physical $\&$ Computational Sciences Directorate, Pacific Northwest National Laboratory, Richland, WA 99352, USA

E-mail: peterpz@uw.edu

\begin{abstract}
Fluoride crystals, due to their low phonon energies, are attractive hosts of trivalent lanthanide ions for applications in upconverting phosphors, quantum information science, and solid-state laser refrigeration. In this article, we report the rapid, lowcost hydrothermal synthesis of potassium lutetium fluoride (KLF) microcrystals for applications in solid-state laser refrigeration. Four crystalline phases were synthesized, namely orthorhombic $\mathrm{K}_{2} \mathrm{LuF}_{5}$ (Pnma), trigonal $\mathrm{KLuF}_{4}\left(\mathrm{P}_{1} 21\right)$, orthorhombic
\end{abstract}


$\mathrm{KLu}_{2} \mathrm{~F}_{7}$ (Pna2 $\left.2_{1}\right)$, and cubic $\mathrm{KLu}_{3} \mathrm{~F}_{10}(\mathrm{Fm} \overline{3} \mathrm{~m})$, with each phase exhibiting unique microcrystalline morphologies. Luminescence spectra and emission lifetimes of the four crystalline phases were characterized based on the point-group symmetry of trivalent cations. Laser refrigeration was measured by observing both the optomechanical eigenfrequencies of microcrystals on cantilevers in vacuum, and also the Brownian dynamics of optically trapped microcrystals in water. Among all four crystalline phases, the most significant cooling was observed for $10 \% \mathrm{Yb}_{\mathrm{KLuF}}$ with cooling of $8.6 \pm 2.1 \mathrm{~K}$ below room temperature. Reduced heating was observed with $10 \% \mathrm{Yb}: \mathrm{K}_{2} \mathrm{LuF}_{5}$

\section{Keywords}

laser refrigeration, hydrothermal synthesis, rare-earth doped crystals, opto-mechanical thermometry, cold Brownian motion

\section{Introduction}

Rare-earth (RE) doped materials have been investigated for use in a wide range of applications including bioimaging, ${ }^{1}$ photocatalysis, ${ }^{2}$ solar cells,${ }^{3-5}$ telecommunications,${ }^{6}$ quantum information science, ${ }^{7}$ and laser refrigeration, also known as solid-state laser cooling. ${ }^{8}$ Laser cooling in solids arises from the extraction of thermal energy stored in phonon modes of the solid lattice by a low-entropy laser. The extraction of heat from the solid happens as a result of spontaneous, anti-Stokes luminescence following the excitation of ions. The thermal energy is converted to the additional energy required for anti-Stokes emission. The anti-Stokes photons that escape from the solid transfer thermal energy out of the material. This phenomenon was first proposed by Pringsheim in $1929 .{ }^{9}$ Later in 1950, Kastler proposed the use of RE ions for anti-Stokes laser refrigeration because of their high quantum efficiency. ${ }^{10}$ The first experimental demonstration of solid-state laser cooling involved a Yb-doped ZBLANP glass material in $1995 .{ }^{11}$ To date, net cooling has been observed in a 
wide variety of glasses ${ }^{11-15}$ and crystals $^{15-18}$ doped with RE ions. Most recently, Yb-doped lithium yttrium fluoride $\left(\mathrm{LiYF}_{4}\right)$ was cooled to cryogenic temperatures $(91 \mathrm{~K})$ through a vibration-free anti-Stokes photoluminescence process. ${ }^{19-22}$

Currently, the development of new laser refrigeration materials is focused primarily on RE-doped ceramics ${ }^{23}$ due to stringent requirements for near-unity radiative quantum efficiencies and low background absorption coefficients. The high quantum efficiency arises from the unique $4 f$ orbitals of RE ions, which are shielded from local perturbations by $5 s$ and $5 p$ electrons. Ytterbium is the most common dopant for anti-Stokes laser refrigeration materials due to its relatively high energy gap (ca. $1 \mathrm{eV}$ ) between ground and first excited (f-f) states and its lack of higher-energy excited states. Because non-radiative decay rates are inversely proportional to the energy gap between the excited and ground states ${ }^{24}$ the relatively large gap of $\mathrm{Yb}$ helps suppress non-radiative relaxation and hence lattice heating. The lack of higher-energy excited states avoids detrimental heating based on excited-state absorption followed by exothermic downconversion. From a practical standpoint, the $1 \mathrm{eV}$ $f-f$ energy gap also coincides with the energies of many commercial lasers. Among all host materials, fluoride crystals are especially attractive candidates for solid-state laser cooling because they are transparent in wide spectral regions and exhibit only low phonon energies, which minimizes background absorption and the non-radiative losses, and they are chemically hard. Combinations of $\mathrm{Yb}^{3+}$ dopants with fluoride hosts are thus good candidates for laser cooling materials.

Beyond $\mathrm{LiYF}_{4}$, advances are quickly being made with other host materials within the alkali-RE-fluoride family. Recently, $\mathrm{NaYF}_{4}$ nanowires were reported to achieve internal laser refrigeration within an aqueous solution. ${ }^{25}$ Laser refrigeration of bulk Czochralski-grown $\mathrm{KYF}_{4}$ has also been observed. ${ }^{26}$ Within the alkali-RE-fluoride family, potassium-based materials have been studied less thoroughly than their $\mathrm{Na}$ and Li counterparts, due in part to the complexity of the potassium-RE-fluoride phase diagram. ${ }^{27}$ The investigation of potassiumRE-fluoride systems started over half a century ago ${ }^{28}$ and five stable crystallographic phases 
are known including $\mathrm{K}_{3} \mathrm{REF}_{6}, \mathrm{~K}_{2} \mathrm{REF}_{5}, \mathrm{KREF}_{4}, \mathrm{KRE}_{2} \mathrm{~F}_{7}$, and $\mathrm{KRE}_{3} \mathrm{~F}_{10}{ }^{27,29}$ This multitude of phases can been represented by writing the stoichiometric formulae in a binary form as $(\mathrm{KF})_{\mathrm{x}} \cdot\left(\mathrm{REF}_{3}\right)_{\mathrm{y}}$. Changing the $\mathrm{KF} / \mathrm{REF}_{3}$ binary compound ratio results in different crystal structures. This observation has attracted interest in potassium-RE-fluorides and their applications in laser refrigeration, as other members in this family, such as $\mathrm{LiYF}_{4}$ (tetragonal phase) and $\mathrm{NaYF}_{4}(\alpha$-cubic and $\beta$-hexagonal phases), are known to have only one stoichiometry. This variety of crystal structures provides a rich array of potential host environments for $\mathrm{Yb}^{3+}$ ions for laser refrigeration. Beyond the diversity of phases, recent work has also explored replacing the common host element yttrium with lutetium, ${ }^{30}$ as the lutetium-lithium-fluoride phase diagram exhibits congruent melting. ${ }^{31}$ There is also a close match in cation size between $\mathrm{Lu}^{3+}(861 \mathrm{pm})$ and the dopant ion $\mathrm{Yb}^{3+}(868 \mathrm{pm}),{ }^{32}$ which allows better crystal quality.

In this work we report the rapid, low-cost hydrothermal synthesis of four $(\mathrm{KF})_{\mathrm{x}} \cdot\left(\mathrm{REF}_{3}\right)_{\mathrm{y}}$ crystallographic phases $\left(\mathrm{KLu}_{3} \mathrm{~F}_{10}, \mathrm{KLu}_{2} \mathrm{~F}_{7}, \mathrm{KLuF}_{4}\right.$, and $\left.\mathrm{K}_{2} \mathrm{LuF}_{5}\right)$, some of which are challenging to grow with bulk growth methods. ${ }^{27}$ Each crystalline phase exhibits a distinct morphology, with crystallite sizes ranging from nanometers to hundreds of micrometers. Photoluminescence spectra and lifetimes of the four crystalline phases were characterized based on the point-group symmetry of trivalent cations in each phase. $\mathrm{Yb}(\mathrm{III})$ lifetimes in $\mathrm{KLuF}_{4}$ and $\mathrm{K}_{2} \mathrm{LuF}_{5}$ were measured to be above $2 \mathrm{~ms}$, primarily due to a combination of their relatively weak oscillator strength ${ }^{27}$ and effects arising from radiation trapping. It is beneficial to use laser hosts with long luminescence lifetimes for energy storage and Q-switching. ${ }^{27}$ The laser-refrigeration performance of each phase was examined via (i) opto-mechanical eigenfrequencies of the crystals on cantilevers in vacuum and/or (ii) the Brownian dynamics of crystals optically trapped in water. Micron-scale $\mathrm{Yb}: \mathrm{KLuF}_{4}$ shows the most significant cooling among all four crystalline phases investigated, with minimum temperatures of $8.6 \pm$ $2.1 \mathrm{~K}$ below room temperature. This is the first experimental demonstration of laser refrigeration using hydrothermally grown potassium lutetium fluoride in either aqueous media or 
vacuum. This material platform is promising for future use in micron-scale radiation balanced lasers, in which cooling by anti-Stokes luminescence compensates the heat generated during lasing. ${ }^{33}$

\section{Results and discussion}

a

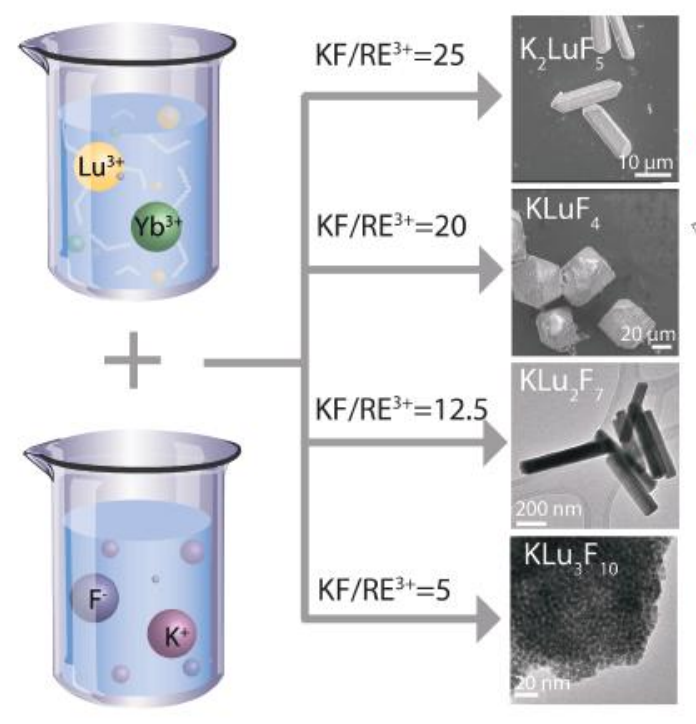

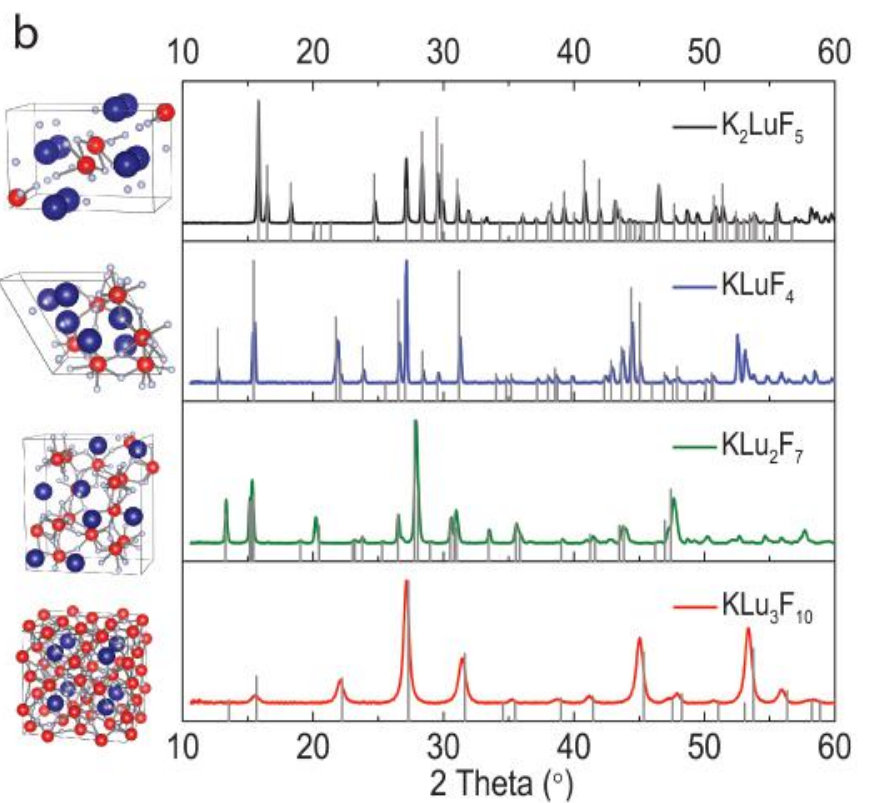

Figure 1: a) Microscopic images of KLF crystals synthesized with various molar ratios of $\mathrm{KF}$ to $\mathrm{RE}^{3+}$. The counter anion is $\mathrm{NO}_{3}{ }^{-}$for all syntheses. b) XRD patterns of KLF samples synthesized with $\mathrm{KF} / \mathrm{RE}=25,20,12.5,5$ (from top to bottom) and the corresponding lattice structures. The blue, red, and grey atoms represent potassium, lutetium, and fluorine, respectively. The standard data of $\mathrm{K}_{2} \mathrm{LuF}_{5}$ (PDF Card 04-005-6013), $\mathrm{KLuF}_{4}$ (PDF 04-0194298), $\mathrm{KYb}_{2} \mathrm{~F}_{7}$ (PDF 00-027-0459, $\mathrm{KLu}_{3} \mathrm{~F}_{10}$ (PDF 04-002-4130) are given as references. $\mathrm{KLu}_{2} \mathrm{~F}_{7}$ is not available in the database. Its XRD patterns are shifted slightly to larger angles with respect to the $\mathrm{KYb}_{2} \mathrm{~F}_{7}$ reference because the $\mathrm{Lu}^{3+}$ ionic radius $(0.861 \AA)$ is slightly smaller than that of $\mathrm{Yb}^{3+}(0.868 \AA) .{ }^{32}$

Rapid, low-cost hydrothermal methods were used to grow Yb-doped $(\mathrm{KF})_{\mathrm{x}} \cdot\left(\mathrm{LuF}_{3}\right)_{\mathrm{y}}$ crystals. Four phases, $\mathrm{KLu}_{3} \mathrm{~F}_{10}, \mathrm{KLu}_{2} \mathrm{~F}_{7}, \mathrm{KLuF}_{4}$, and $\mathrm{K}_{2} \mathrm{LuF}_{5}$, were successfully synthesized by varying the concentration of the KF precursor (See SI for details). Figure 1 shows the morphologies and powder X-ray diffraction (XRD) patterns of these four phases at different $\mathrm{KF} / \mathrm{RE}^{3+}$ ratios, from 5:1 to 25:1. The as-synthesized samples shown in Figure 1 exhibit dis- 
tinct shapes and lattice structures. Under otherwise identical reaction conditions, increasing the $\mathrm{KF} / \mathrm{RE}^{3+}$ ratio increases the sizes of the resulting particles. Furthermore, the characteristic morphology of the particles changes as shown in Figure 1 . The $\mathrm{K}_{3} \mathrm{LuF}_{6}$ phase was not observed using hydrothermal synthesis, even with a $\mathrm{KF} / \mathrm{RE}^{3+}$ ratio as high as 60 . This observation is consistent with previous experiments that show only four phases of $(\mathrm{KF})_{\mathrm{x}} \cdot\left(\mathrm{YbF}_{3}\right)_{\mathrm{y}}$ crystals in hydrothermal synthesis. ${ }^{34}$ The diffraction patterns of these products prepared with different $\mathrm{KF} / \mathrm{RE}$ ratios can be indexed to orthorhombic $\mathrm{K}_{2} \mathrm{LuF}_{5}$, trigonal $\mathrm{KLuF}_{4}$, orthorhombic $\mathrm{KLu}_{2} \mathrm{~F}_{7}$, and cubic $\mathrm{KLu}_{3} \mathrm{~F}_{10}$. More details of these four crystallographic phases are listed in Table 1. Among these phases, $\mathrm{KLu}_{2} \mathrm{~F}_{7}$ and $\mathrm{KLuF}_{4}$ are non-centrosymmetric, which motivates the potential application of these materials for non-linear optics.

Table 1: Crystallographic information for $\mathrm{K}_{2} \mathrm{LuF}_{5}, \mathrm{KLuF}_{4}, \mathrm{KLu}_{2} \mathrm{~F}_{7}$, and $\mathrm{KLu}_{3} \mathrm{~F}_{10}$

\begin{tabular}{|c|c|c|c|c|c|}
\hline Sample & Crystal System & $\begin{array}{l}\text { Space } \\
\text { Group }\end{array}$ & $\begin{array}{l}\mathrm{Yb}^{3+} \text { Site } \\
\text { Symmetry }\end{array}$ & Lattice Parameter & Centro / Noncentro \\
\hline $\mathrm{K}_{2} \mathrm{LuF}_{5}$ & Orthorhombic & Pnma & $C_{2}$ & $\begin{array}{c}\mathrm{a}=10.699 \AA, \mathrm{b}=6.534 \AA \\
\mathrm{c}=7.145 \AA\end{array}$ & Centrosymmetric \\
\hline $\mathrm{KLuF}_{4}$ & Trigonal & $P 3_{1} 21$ & $C_{1}$ & $\begin{array}{c}\mathrm{a}=\mathrm{b}=8.012 \AA, \mathrm{c}=10.058 \AA \\
\left(\alpha=\beta=90^{\circ}, \gamma=120^{\circ}\right)\end{array}$ & Noncentrosymmetric \\
\hline $\mathrm{KLu}_{2} \mathrm{~F}_{7}$ & Orthorhombic & $P n a 2_{1}$ & $C_{2}, C_{1}$ & $\begin{array}{c}\mathrm{a}=11.716 \AA, \mathrm{b}=13.241 \AA \\
\mathrm{c}=7.739 \AA\end{array}$ & Noncentrosymmetric \\
\hline $\mathrm{KLu}_{3} \mathrm{~F}_{10}$ & Cubic & $F m \overline{3} m$ & $C_{4 v}$ & $\mathrm{a}=\mathrm{b}=\mathrm{c}=11.305 \AA$ & Centrosymmetric \\
\hline
\end{tabular}

Control over crystallographic phases and their corresponding morphologies requires a deep understanding of crystal growth. Among the various $\mathrm{KLF}$ crystal systems, $\mathrm{K}_{2} \mathrm{LuF}_{5}$ is a good candidate to study crystallization kinetics in nanometer- and micron size-scales because of its rod-like morphology and high growth speed. The growth velocity of $\mathrm{K}_{2} \mathrm{LuF}_{5}$ is at least $0.77 \mu \mathrm{m} / \mathrm{min}$ along the growth direction and $0.10 \mu \mathrm{m} / \mathrm{min}$ perpendicular to the growth direction in the first hour (See Supporting Information for details). We also observed that the $\mathrm{Yb}^{3+}$ concentration controls the length of $\mathrm{K}_{2} \mathrm{LuF}_{5}$ rods, with that length increases with higher $\mathrm{Yb}^{3+}$ concentrations. (See Supporting Information for details)

Figure 2 summarizes the photoluminescence (PL) of $10 \% \mathrm{Yb}: \mathrm{K}_{2} \mathrm{LuF}_{5}, 10 \% \mathrm{Yb}: \mathrm{KLuF}_{4}$, 10\% Yb:KLu $\mathrm{K}_{7}$ and $10 \% \mathrm{Yb}: \mathrm{KLu}_{3} \mathrm{~F}_{10}$ upon $1020 \mathrm{~nm}$ laser excitation. $\mathrm{Yb}^{3+}$ in the KLF host 

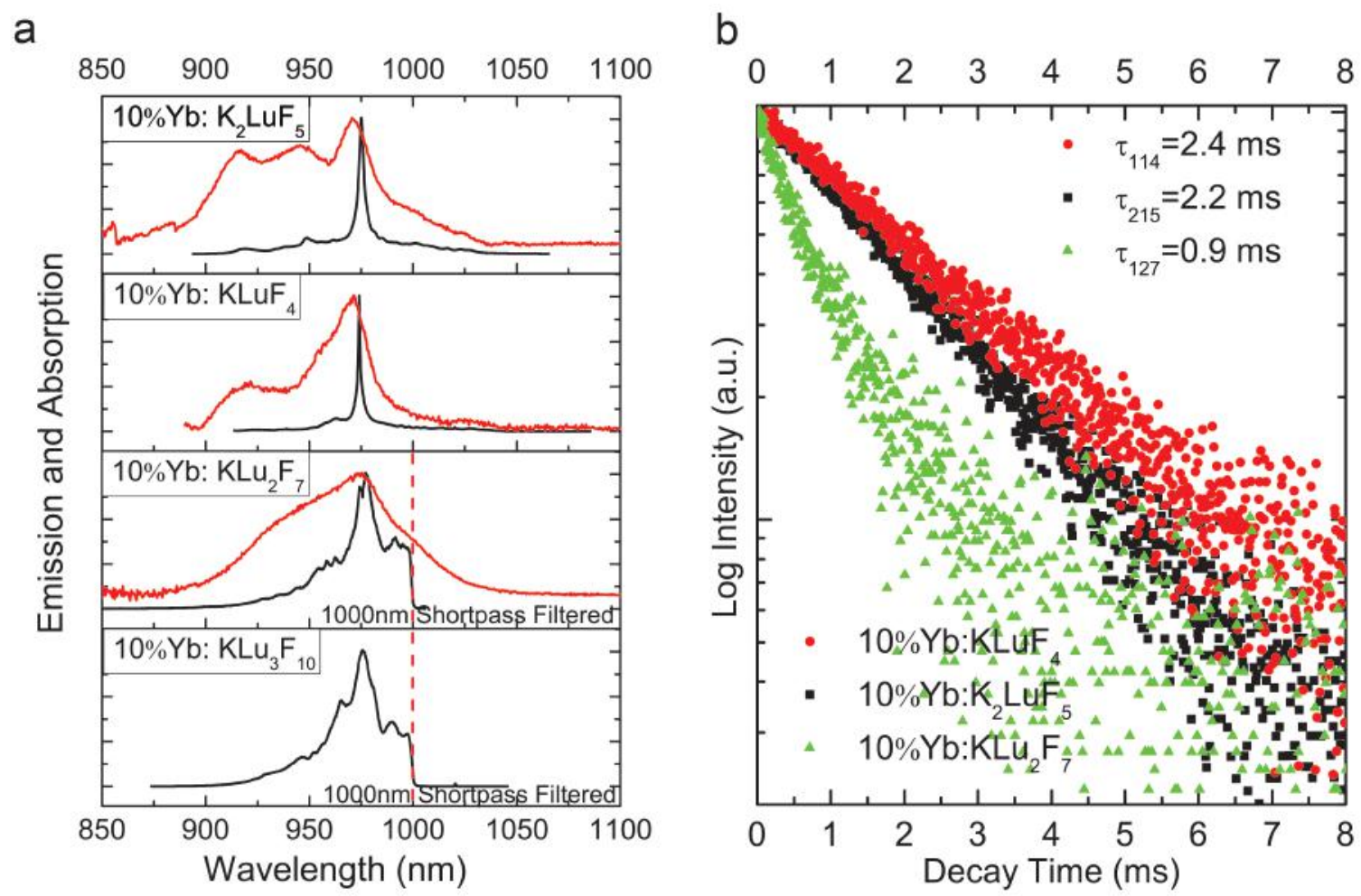

Figure 2: Room-temperature PL (black) and absorption (red) spectra of probing the $\mathrm{Yb}$ (III) ${ }^{2} \mathrm{~F}_{5 / 2} \rightarrow{ }^{2} \mathrm{~F}_{7 / 2}$ electronic transition of $10 \% \mathrm{Yb}: \mathrm{K}_{2} \mathrm{LuF}_{5}, 10 \% \mathrm{Yb}: \mathrm{KLuF}_{4}, 10 \% \mathrm{Yb}: \mathrm{KLu}_{2} \mathrm{~F}_{7}$ and $10 \% \mathrm{Yb}: \mathrm{KLu}_{3} \mathrm{~F}_{10}$ under $1020 \mathrm{~nm}$ laser excitation. b) PL decay profiles of the $\mathrm{Yb}(\mathrm{III}){ }^{2} \mathrm{~F}_{5 / 2}$ $\rightarrow{ }^{2} \mathrm{~F}_{7 / 2}$ transitions of $10 \% \mathrm{Yb}_{\mathrm{K}_{2}} \mathrm{LuF}_{5}, 10 \% \mathrm{Yb}: \mathrm{KLuF}_{4}$ and $10 \% \mathrm{Yb}: \mathrm{KLu}_{2} \mathrm{~F}_{7}$. The fitted luminescence lifetimes for each sample are provided. 
matrix has its ${ }^{2} F_{7 / 2}$ ground-state and ${ }^{2} F_{5 / 2}$ excited-state manifolds split into Stark levels due to the electrostatic crystal field. If the $\mathrm{Yb}^{3+}$ point symmetry is lower than cubic $(O$, $\left.O_{h}, T_{d}\right)$, the ${ }^{2} F_{5 / 2}$ and ${ }^{2} F_{7 / 2}$ states split into 3 and 4 Kramers doubly-degenerate Stark levels, respectively, otherwise they split into 2 and 3 crystal-field levels, respectively. ${ }^{35}$ The Kramers degeneracies are only lifted under an applied magnetic field. The Stark multiplet structure of excited and ground states determines the relaxation channels resulting in Stokes and anti-Stokes emission. ${ }^{36}$ In Figure 2, the emission spectra of each sample are distinct due to differences in $\mathrm{Yb}$ site symmetries. The full emission spectra of $\mathrm{Yb}^{3+}{ }^{2} F_{7 / 2} \rightarrow{ }^{2} F_{7 / 2}$ transitions for $10 \% \mathrm{Yb}: \mathrm{K}_{2} \mathrm{LuF}_{5}$ and $10 \% \mathrm{Yb}: \mathrm{KLuF}_{4}$ single crystals were recorded by using time-gated spectroscopy. Although there are twelve allowed transitions, only one dominant peak around $974 \mathrm{~nm}$ was observed. For the $10 \% \mathrm{Yb}: \mathrm{KLuF}_{4}$ sample, the full width at half maximum (FWHM) of the primary peak is less than $2 \mathrm{~nm}$ without any further processing of the crystal. A side peak around $1030 \mathrm{~nm}$ is reported in structurally similar $\mathrm{Yb}: \mathrm{KYF}_{4},{ }^{26}$ but we did not observe such peaks in our detector range. Besides the intense emission at $974 \mathrm{~nm}, 10 \% \mathrm{Yb}: \mathrm{K}_{2} \mathrm{LuF}_{5}$ and $10 \% \mathrm{Yb}: \mathrm{KLuF}_{4}$ both have luminescence lifetimes longer than 2 ms (Figure 2b). For $10 \% \mathrm{Yb}: \mathrm{KLu}_{2} \mathrm{~F}_{7}$ and $10 \% \mathrm{Yb}: \mathrm{KLu}_{3} \mathrm{~F}_{10}$, the measured $\mathrm{Yb}$ lifetimes are short compared to the other samples; $10 \% \mathrm{Yb}: \mathrm{KLu}_{2} \mathrm{~F}_{7}$ has a luminescence lifetime of $874 \mu \mathrm{s}$ and for $10 \% \mathrm{Yb}: \mathrm{KLu}_{3} \mathrm{~F}_{10}$ the luminescence lifetime was shorter than the $100 \mu$ s resolution of our detector. Due to the relatively short luminescence lifetime, the time-gated method for a full-range emission spectrum did not work well for the $10 \% \mathrm{Yb}: \mathrm{KLu}_{2} \mathrm{~F}_{7}$ and $10 \% \mathrm{Yb}: \mathrm{KLu}_{3} \mathrm{~F}_{10}$ samples. Instead, a $1000 \mathrm{~nm}$ short-pass filter was applied to block the laser line. The spectra below $1000 \mathrm{~nm}$ were recorded for $10 \% \mathrm{Yb}: \mathrm{KLu}_{2} \mathrm{~F}_{7}$ and $10 \% \mathrm{Yb}: \mathrm{KLu}_{3} \mathrm{~F}_{10}$. According to Figure 2a, the PL from these two crystalline phases are much broader than the previous two phases. Such differences in spectral lineshape result from differences in the local symmetry and strength of the electrostatic crystal field, ${ }^{37}$ as well as from differences in inhomogeneous broadening.

The lifetime we measured is the inverse of the total decay rate constant, $W=W_{r}+W_{n r}$, 
which has radiative $\left(W_{r}\right)$ and nonradiative $\left(W_{n r}\right)$ components. The radiative decay rate constant reflects the oscillator strength of the same transition and is related to the local symmetry of the $\mathrm{Yb}^{3+}$, whereas the nonradiative decay rate constant is affected by the lattice phonon energies, dopant concentrations, and defects with high-energy vibrational modes. Radiative and nonradiative relaxation mechanisms can be modified by the local symmetry of the host lattice and by crystal size, respectively. ${ }^{38}$ Here the radiative decay rate constant cannot be estimated without more detailed spectroscopic data. Some potential reasons for the lifetime difference can be ruled out, such as impurities inside the crystal, energy transfer processes that depend on dopant concentration, and lattice phonon energies. In our study, the starting materials, synthesis method, and Yb-doping concentrations of these KLF crystals are all the same. Instead, the long emission lifetimes of $10 \% \mathrm{Yb}: \mathrm{K}_{2} \mathrm{LuF}_{5}$ and $10 \% \mathrm{Yb}_{\mathrm{KLuF}_{4}}$ are attributed to the low nonradiative decay rate and also potential radiation trapping in these large-size crystals. ${ }^{39}$ Surface defects are one of the main causes of non-radiative decay for these fluoride crystals as the fluoride phonon energy is low. FTIR spectra with the highest vibrational mode of each crystalline phase are shown in Figure S8. A large crystal volume significantly lowers the surface-to-volume ratio and thus decreases nonradiative decay. ${ }^{40} \mathrm{Yb}^{3+}$ ions exhibit significant overlap between their absorption and emission spectra. Therefore, the reabsorption of emission is non-negligible in crystals with characteristic dimensions on the order of 100 micrometers. This can result in a measured luminescence lifetimes as much as $30 \%$ longer than the expected value. ${ }^{41}$ In this approximation, the corrected lifetimes of $10 \% \mathrm{Yb}: \mathrm{K}_{2} \mathrm{LuF}_{5}$ and $10 \% \mathrm{Yb}: \mathrm{KLuF}_{4}$ are $1.68 \mathrm{~ms}$ and $1.86 \mathrm{~ms}$, respectively. ${ }^{41}$ The sharp and intense emission at $974 \mathrm{~nm}$ for these two samples is possibly the result of reabsorption. Such luminescence reabsorption is an important factor for laser media, as it can be used as an additional pumping source, recycling energy that would otherwise be lost as spontaneous luminescence. Reabsorption can therefore have a substantial influence on laser efficiency. ${ }^{39}$ Because $W_{n r}$ is temperature dependent, the PL lifetime could be used to probe local temperature at the nanometer scale. ${ }^{42}$ 


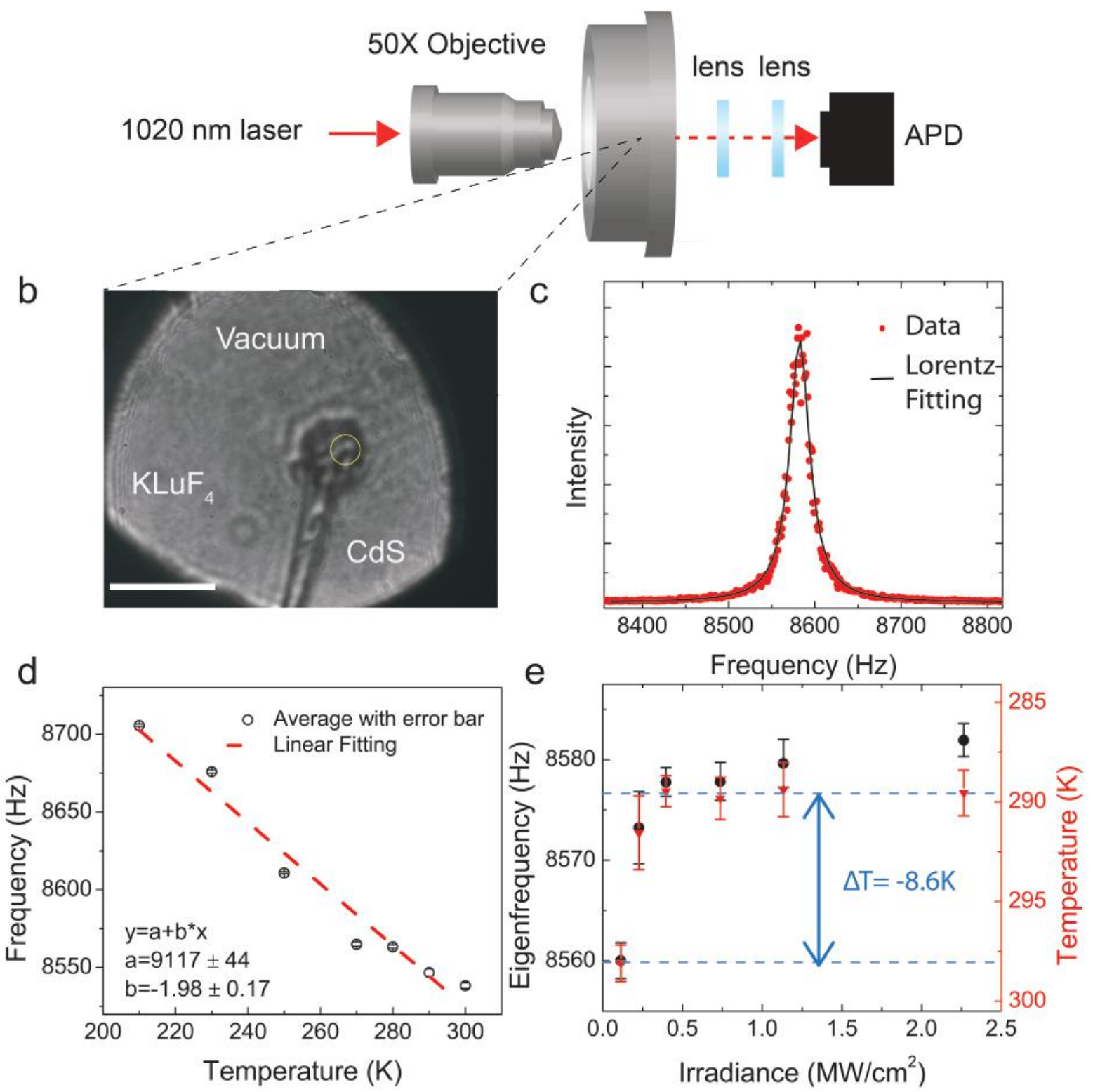

Figure 3: a) Schematic of the experimental setup for the eigenfrequency measurement. b) A microscopic image of a CdSNR with a $10 \%: \mathrm{KLuF}_{4}$ crystal. scale bar: $10 \mu \mathrm{m} \mathrm{c}$ ) A representative Lorentzian fitting curve (black) of the eigenfrequency peak in the Fourier transformed signal from the APD (red). d) Frequency vs. temperature calibration of the CdSNR + crystal cantilever. Black open circle: average frequency of six measurements. The error bar represents one standard deviation. Red: linear least-squares fit. The eigenfrequency decreases when increasing the temperature. e) The frequencies measured (black) at various irradiances and the frequencies after subtracting the effect of optical trapping (red). The subtracted frequencies are used to fit the temperature. 
To evaluate the laser refrigeration properties of these as-synthesized crystals, the crystals were transferred onto a cadmium sulfide nanoribbon cantilever (CdSNR) with low NIR absorption to measure the temperature via the cantilever's optomechanical eigenfrequency. ${ }^{43,44}$ As the temperature decreases, the Young's modulus of the CdSNR increases and thus the eigenfrequency increases as well. The experimental setup and an optical micrograph of a $10 \% \mathrm{Yb}: \mathrm{KLuF}_{4}$ crystal on the CdSNR in a vacuum cryostat are shown in Figure 3a and Figure 3b. Details for device fabrication are discussed further in the experimental section. Figure 3c shows a representative eigenfrequency spectrum fit numerically with a Lorentzian. The eigenfrequencies at various temperatures were measured and linearly fit (Figure 3d) with a slope of $2 \mathrm{~Hz} / \mathrm{K}$ for calibration of subsequent laser refrigeration experiments. When excited by a $1020 \mathrm{~nm}$ laser, the eigenfrequency was observed to increase with increasing laser irradiance, indicating net cooling of the cantilever. The eigenfrequency blue-shifts $22 \pm 3$ $\mathrm{Hz}$ at the maximum irradiance (Figure 3e).

The eigenfrequency of the cantilever depends not only on its temperature-dependent Young's modulus, but also on potential laser trapping forces, where the laser trap can be modeled as a Hookean spring located at the end of the cantilever. With increased laser irradiance, the spring constant increases and thus the cantilever eigenfrequency increases. The red data in Figure 3e show that after subtracting the increase in eigenfrequency related to optical trapping, the blue-shift due to the temperature change is $17 \pm 4 \mathrm{~Hz}$ (see Figure S6 and discussion in Supporting Information). According to the temperature calibration, this shift indicates a temperature decrease of $8.6 \pm 2.1 \mathrm{~K}$ (Figure 3e) for $10 \% \mathrm{Yb}: \mathrm{KLuF}_{4}$. Laser refrigeration is not observed in other crystal phases (see Supporting Information).

In addition to laser refrigeration in vacuum, we observed laser refrigeration of $10 \% \mathrm{Yb}: \mathrm{KLuF}_{4}$ in water as well. A home-built laser trapping instrument illustrated in Figure 4a was used to measure the temperature of an individual $10 \% \mathrm{Yb}: \mathrm{KLuF}_{4}$ microcrystal through the analysis of the particle's cold Brownian motion at a trapping wavelength of $\lambda=1020 \mathrm{~nm}$. To minimize heating of the trapping chamber the microcrystals were trapped at a distance of 
$40 \mu \mathrm{m}$ away from the quartz substrate, approximately in the middle of the fluid chamber. The dynamics of a trapped particle were observed by recording the forward-scattered laser light via a quadrant photodiode (QPD). A power spectral density (PSD) vs. frequency plot (Figure 4b) was generated by performing the Fourier transform. The temperature was extracted from the PSD by the method in Refs. ${ }^{25,45}$ In brief, a $32 \mathrm{~Hz}$ oscillation of the trapping chamber was driven by the piezo-stage to convert the photovoltage signal in units of volts to units of length $[\mathrm{m}]$. The local temperature of the trapped $10 \% \mathrm{Yb}: \mathrm{KLuF}_{4}$ microcrystal and surrounding water decreased $7.2 \pm 0.6 \mathrm{~K}$ below room temperature as shown in Figure 4c. As a control, we also measured the temperature of the same $10 \% \mathrm{Yb}: \mathrm{KLuF}_{4}$ microcrystal trapped in water with a $1064 \mathrm{~nm}$ laser, which is not resonant with $\mathrm{Yb}^{3+}$ absorption but can heat the surrounding water. With the same temperature extraction method, the sample temperature increased above $50^{\circ} \mathrm{C}$. Optical trapping experiments with the $10 \% \mathrm{Yb}: \mathrm{K}_{2} \mathrm{LuF}_{5}$ phase showed reduced heating in water as evidenced by stable temperature with increased irradiance (Figure S7). Surface defects could be the reason for different cooling performances of these different KLF phases. Small crystals with large surface-to-volume ratios have large nonradiative losses, especially for the $\mathrm{KLu}_{3} \mathrm{~F}_{10}$ nanoparticles, which shows a short luminescence lifetime. Besides the size effect, the maximal phonon energies of $\mathrm{KLu}_{3} \mathrm{~F}_{10}$ and $\mathrm{KLu}_{2} \mathrm{~F}_{7}$ are slightly higher than those of $\mathrm{KLuF}_{4}$ and $\mathrm{K}_{2} \mathrm{LuF}_{5}$, which facilitates nonradiative relaxation. The PL linewidth and site homogeneity are also important for laser refrigeration. Low temperature spectroscopic studies of these factors will be the subject of future work.

\section{Conclusions}

Potassium lutetium fluoride is a relatively unexplored member of the alkali-RE-fluoride family for solid-state laser refrigeration. It has several crystallographic polymorphs with various $\mathrm{KF} / \mathrm{LuF}_{3}$ binary compound ratios, providing a rich array of potential host environments for $\mathrm{Yb}^{3+}$ ions. In this article we present the low-cost, rapid hydrothermal synthesis of 

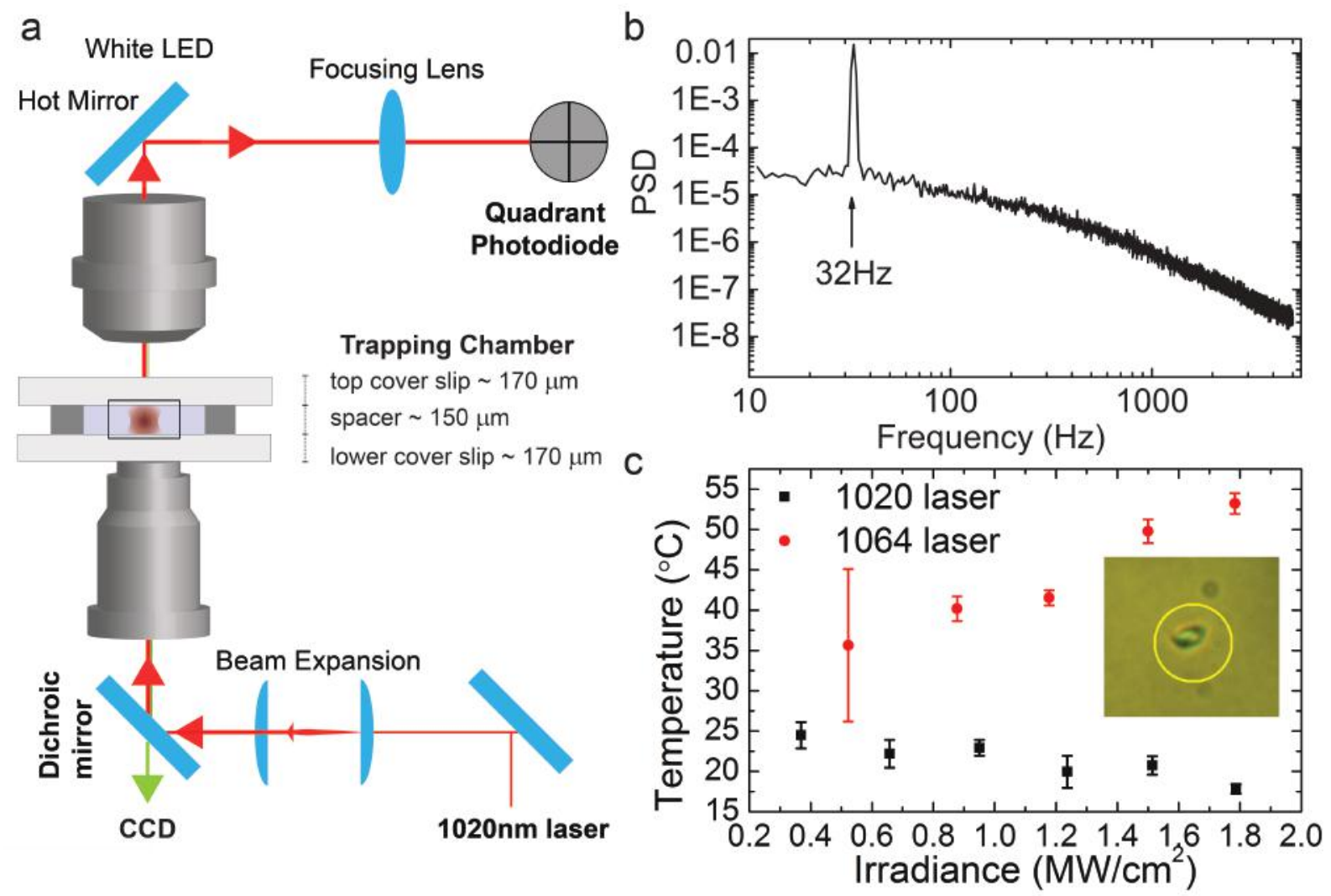

Figure 4: a) Schematic of laser-trapping setup. b) A representative power spectrum of a trapped particle undergoing Brownian motion in water with $32 \mathrm{~Hz}$ oscillation. c) The temperature of a $10 \% \mathrm{Yb}: \mathrm{KLuF}_{4}$ crystal trapped by $1020 \mathrm{~nm}$ laser and by $1064 \mathrm{~nm}$ laser in water. 
four distinct phases of KLF nano- or microcrystals with the general stoichiometric formula $(\mathrm{KF})_{\mathrm{x}} \cdot\left(\mathrm{LuF}_{3}\right)_{\mathrm{y}}$, including $\mathrm{KLuF}_{4}$ (trigonal), $\mathrm{KLu}_{2} \mathrm{~F}_{7}$ (orthorhombic), $\mathrm{KLu}_{3} \mathrm{~F}_{10}$ (cubic), and $\mathrm{K}_{2} \mathrm{LuF}_{5}$ (orthorhombic). Each phase was doped with $10 \%$ ytterbium ions, and two of these phases $\left(\mathrm{KLuF}_{4}\right.$ and $\left.\mathrm{K}_{2} \mathrm{LuF}_{5}\right)$ were observed to be viable candidates for solid-state laser refrigeration. Luminescence spectra and lifetimes of the four crystalline phases were characterized and interpreted based on the point-group symmetry of the trivalent cations in each crystal phase. Laser refrigeration was demonstrated using both opto-mechanical cantilevers in vacuum and also through observing the Brownian dynamics of the crystals optically trapped in water. $\mathrm{Yb}: \mathrm{KLuF}_{4}$ shows the most significant cooling, with minimum temperatures $8.6 \pm$ 2.1 K below room temperature. These results provide the first experimental demonstration of laser refrigeration demonstration using KLF materials in any form, including in aqueous medium and vacuum as demonstrated here. Furthermore, each crystalline phase exhibits distinct morphologies that may be advantageous in the future development of micron-scale radiation balanced lasers.

\section{Experimental}

KLF synthesis: Lutetium nitrate $\left(\mathrm{Lu}\left(\mathrm{NO}_{3}\right)_{3}\right)$ and ytterbium nitrate $\left(\mathrm{Yb}\left(\mathrm{NO}_{3}\right)_{3}\right.$ were of 99.99\% purity and purchased from Sigma-Aldrich. Potassium hydroxide (KOH), potassium fluoride $(\mathrm{KF})$, ethanol and oleic acid were analytical grade purchased from Sigma-Aldrich and used without further purification. 18.2 M $\Omega * \mathrm{~cm}$ Milli-Q deionized water was used for each synthesis. For a typical synthesis, $420.8 \mathrm{mg}$ of $\mathrm{KOH}$ was dissolved in $1.5 \mathrm{~mL}$ of water, followed by $5 \mathrm{~mL}$ of ethanol and $5 \mathrm{~mL}$ of oleic acid with stirring. Then, $1.44 \mathrm{~mL}$ of $0.5 \mathrm{M}$ $\mathrm{Lu}\left(\mathrm{NO}_{3}\right)_{3}$ and $0.16 \mathrm{~mL}$ of $0.5 \mathrm{M} \mathrm{Yb}\left(\mathrm{NO}_{3}\right)_{3}$ were added into the mixture. After stirring for $10 \mathrm{~min}, 3 \mathrm{~mL}$ of aqueous KF solution was added dropwise. The molar concentration of the KF solution depends on the target product. After additional agitation for 10 min, the resulting mixture was transferred to a $23 \mathrm{~mL}$ Teflon-lined autoclave and heated at $220^{\circ} \mathrm{C}$ for 
24 hours. After the autoclave cooled to room temperature, crystals were isolated by washing and centrifuging by ethanol and water three times. After drying the product at $60^{\circ} \mathrm{C}$ for 12 hours, they were transferred to a Lindberg Blue tube furnace and heated at $300^{\circ} \mathrm{C}$ for 2 hours to remove the organic surface ligand.

Eigenfrequency measurement: A nanomanipulator (Märzhäuser-Wetzlär) with a tungsten dissecting probe (World Precision Instruments, 100nm) was used to transfer the CdSNR to a silicon wafer. The hydrothermal product was ground to small pieces. Then a ground piece of $10 \% \mathrm{Yb}: \mathrm{KLuF}_{4}$ crystal was transferred to the free end of the cantilever. The silicon substrate loaded with the CdSNR cantilever and the crystal sample was then attached via a copper tape to the stage of an optical cryostat (Janis ST-500). The chamber was pumped down to a pressure of $4.0 \times 10^{-4}$ mbar. Forward-scattered laser radiation was collected with the APD from behind the chamber (Figure 3a). Ten power spectra were averaged to obtain one measurement, as shown in the Figure 3c. The peak then was fit to a Lorentzian profile to obtain the cantilever's eigenfrequency. Temperature calibration was performed by heating the cryostat using a temperature controller (Lake Shore 335, resolution: $10 \mathrm{mK}$ ), and measuring the eigenfrequency of the cantilever at different temperatures using a low laser irradiance $\left(0.1 \mathrm{MW} / \mathrm{cm}^{2}\right)$. For each measurement, an average frequency data point was obtained by fitting six measurements obtained at the same laser power with a gap of 1 min between consecutive measurements. The error bars represent one standard deviation.

Laser trapping and temperature extraction: The laser-tweezer setup was modified based on a modular optical tweezer kit (Thorlabs, OTKB), ${ }^{25}$ where the original condenser lens has been replaced with a 10x Mitutoyo condenser (Plan Apo infinity-corrected long WD objective). The laser was focused by a 100x objective lens (Nikon) to $1.1 \mu \mathrm{m}$. The trapping chamber was a glass slide and coverslip spaced by a double-sided tape (EMS) of $150 \mu \mathrm{m}$ thickness. Microcrystals were trapped at the centre ( 30-40 $\mu \mathrm{m}$ from the surface) of the chamber. The particle Brownian motion traces were collected by the condenser and 
recorded by a quadric photodiode (QPD). The methods for data processing and temperature extraction can be found in refs. ${ }^{25,45}$

\section{Acknowledgement}

X.X., A.P., and P.J.P. acknowledge support from the MURI:MARBLe project under the auspices of the Air Force Office of Scientific Research (Award \#FA9550-16-1-0362). This research was partially supported by the National Science Foundation (NSF) through the UW Molecular Engineering Materials Center, a Materials Research Science and Engineering Center (DMR-1719797, to D.R.G. and P.J.P.). A.B.B and P.J.P. acknowledge support from the U.S. Department of Energy, Office of Science (DOE), Basic Energy Sciences (BES), Division of Materials Sciences and Engineering at the University of Washington (UW) and the Pacific Northwest National Laboratory (PNNL) for TEM and SEM characterization. PNNL is a multiprogram national laboratory operated for DOE by Battelle under Contract No. DEAC05-76RL01830. All authors acknowledge support from the UW Nano-engineered systems institute. Part of this work was conducted at the Molecular Analysis Facility, a National Nanotechnology Coordinated Infrastructure site at the University of Washington which is supported in part by the National Science Foundation (grant NNCI-1542101), the University of Washington, the Molecular Engineering Sciences Institute, and the Clean Energy Institute. We acknowledge N.N. Novikova and S.A. Klimin for their assistance in ATR-FTIR measurements.

\section{Supporting Information Available}

Experimental procedures and characterization data for all samples. Discussion about potential growth mechanisms and crystal growth velocities. 


\section{References}

(1) Kumar, R.; Nyk, M.; Ohulchanskyy, T. Y.; Flask, C. A.; Prasad, P. N. Combined Optical and MR Bioimaging Using Rare Earth Ion Doped $\mathrm{NaYF}_{4}$ Nanocrystals. Adv. Funct. Mater. 2009, 19, 853-859.

(2) Xu, A.-W.; Gao, Y.; Liu, H.-Q. The Preparation, Characterization, and Their Photocatalytic Activities of Rare-Earth-Doped $\mathrm{TiO}_{2}$ Nanoparticles. J. Catal. 2002, 20\%, $151-157$.

(3) Wang, H.-Q.; Batentschuk, M.; Osvet, A.; Pinna, L.; Brabec, C. J. Rare-Earth Ion Doped Up-Conversion Materials for Photovoltaic Applications. Adv. Mater. 2011, 23, $2675-2680$.

(4) Chen, Z.; Zhang, L.; Sun, Y.; Hu, J.; Wang, D. 980-nm Laser-Driven Photovoltaic Cells Based on Rare-Earth Up-Converting Phosphors for Biomedical Applications. Adv. Funct. Mater. 2009, 19, 3815-3820.

(5) Crane, M. J.; Kroupa, D. M.; Gamelin, D. R. Detailed-Balance Analysis of $\mathrm{Yb}^{3+}: \mathrm{CsPb}\left(\mathrm{Cl}_{1-x} \mathrm{Br}_{x}\right)_{3}$ Quantum-Cutting Layers for High-Efficiency Photovoltaics under Real-World Conditions. Energy $\& 3$ Environmental Science 2019, 12, 2486-2495.

(6) Bünzli, J.-C. G.; Eliseeva, S. V. Lanthanide NIR Luminescence for Telecommunications, Bioanalyses and Solar Energy Conversion. J. Rare Earths 2010, 28, 824-842.

(7) Thiel, C. W.; Böttger, T.; Cone, R. L. Rare-Earth-Doped Materials for Applications in Quantum Information Storage and Signal Processing. J. Lumin. 2011, 131, 353-361.

(8) Tarascon, J. M.; McKinnon, W. R.; Greene, L. H.; Hull, G. W.; Vogel,; M., E. Oxygen and Rare-Earth Doping of the 90-K Superconducting PerovskitE $\mathrm{YBa}_{2} \mathrm{Cu}_{3} \mathrm{O}_{7-x}$. Phys. Rev. B 1987, 36, 226-234. 
(9) Pringsheim, P. Zwei Bemerkungen über den Unterschied von Lumineszenz- und Temperaturstrahlung. Zeitschrift für Phys. 1929, 57, 739-746.

(10) Kastler, A. Quelques suggestions concernant la production optique et la détection optique d'une inégalité de population des niveaux de quantifigation spatiale des atomes. Application à l'expérience de Stern et Gerlach et à la résonance magnétique. J. Phys. le Radium 1950, 11, 255-265.

(11) Epstein, R. I.; Buchwald, M. I.; Edwards, B. C.; Gosnell, T. R.; Mungan, C. E. Observation of Laser-Induced Fluorescent Cooling of a Solid. Nature 1995, 37r, 500-503.

(12) Rayner, A.; Friese, M. E. J.; Truscott, A. G.; Heckenberg, N. R.; Rubinsztein-dunlop, H. Laser Cooling of a Solid from Ambient Temperature. J. Mod. Opt. 2001, 48, 103-114.

(13) Hoyt, C. W.; Sheik-Bahae, M.; Epstein, R. I.; Edwards, B. C.; Anderson, J. E. Observation of Anti-Stokes Fluorescence Cooling in Thulium-Doped Glass. Phys. Rev. Lett. 2000, 85, 3600-3603.

(14) Fernández, J.; Mendioroz, A.; García, A.; Balda, R.; Adam, J. Anti-Stokes LaserInduced Internal Cooling of Yb-doped Glasses. Phys. Rev. B - Condens. Matter Mater. Phys. 2000, 62, 3213-3217.

(15) Fernandez, J.; Garcia-Adeva, A. J.; Balda, R. Anti-Stokes Laser Cooling in Bulk Erbium-Doped Materials. Phys. Rev. Lett. 2006, 97, 033001.

(16) Epstein, R. I.; Brown, J. J.; Edwards, B. C.; Gibbs, A. Measurements of Optical Refrigeration in Ytterbium-Doped Crystals. J. Appl. Phys. 2001, 90, 4815-4819.

(17) Mendioroz, A.; Fernández, J.; Voda, M.; Al-Saleh, M.; Balda, R.; García-Adeva, A. J. Anti-Stokes Laser Cooling in $\mathrm{Yb}^{3+}$-Doped $\mathrm{KPb}_{2} \mathrm{Cl}_{5}$ Crystal. Opt. Lett. 2002, $27,1525$.

(18) Bigotta, S.; Parisi, D.; Bonelli, L.; Toncelli, A.; Tonelli, M.; Di Lieto, A. Spectroscopic 
and Laser Cooling Results on $\mathrm{Yb}^{3+}$-Doped $\mathrm{BaY}_{2} \mathrm{~F}_{8}$ Single Crystal. J. Appl. Phys. 2006, 100, 013109.

(19) Seletskiy, D. V.; Melgaard, S. D.; Bigotta, S.; Di Lieto, A.; Tonelli, M.; Sheik-Bahae, M. Laser Cooling of Solids to Cryogenic Temperatures. Nat. Photonics 2010, 4, 161-164.

(20) Seletskiy, D. V.; Melgaard, S. D.; Epstein, R. I.; Di Lieto, A.; Tonelli, M.; SheikBahae, M. Local Laser Cooling of Yb:YLF to 110 K. Opt. Express 2011, 19, 18229.

(21) Melgaard, S. D.; Seletskiy, D. V.; Di Lieto, A.; Tonelli, M.; Sheik-Bahae, M. Optical Refrigeration to 119 K, Below National Institute of Standards and Technology cryogenic Temperature. Opt. Lett. 2013, 38, 1588.

(22) Melgaard, S. D.; Albrecht, A. R.; Hehlen, M. P.; Sheik-Bahae, M. Solid-State Optical Refrigeration to Sub-100 Kelvin Regime. Sci. Rep. 2016, 6, 20380.

(23) Sheik-Bahae, M.; Epstein, R. I. Optical Refrigeration. Nat. Photonics 2007, 1, 693-699.

(24) Riseberg, L. A.; Moos, H. W. Multiphonon Orbit-Lattice Relaxation of Excited States of Rare-earth Ions in Crystals. Phys. Rev. 1968, 174, 429-438.

(25) Zhou, X.; Smith, B. E.; Roder, P. B.; Pauzauskie, P. J. Laser Refrigeration of Ytterbium-Doped Sodium-Yttrium-Fluoride Nanowires. Adv. Mater. 2016, 28, 86588662.

(26) Volpi, A.; Cittadino, G.; Di Lieto, A.; Tonelli, M. Anti-Stokes Cooling of Yb-doped $\mathrm{KYF}_{4}$ Single Crystals. J. Lumin. 2018, 203, 670-675.

(27) Chai, B. H. T.; Lefaucheur, J.; Pham, A.-T.; Loutts, G. B.; Nicholls, J. F. Growth of High-Quality Single Crystals of KYF 4 by TSSG Method. 1993; pp 131-135.

(28) Bukhalova, G.; Bahaeva, E.; TM, K. Phase Diagrams for Ceramists. Zh. Neorg. Khim 1965, 10, 2127-2131. 
(29) Levin, E.M.; McMurdie, H. Am. Ceram. Soc.; American Ceramic Society, Inc.,Columbus, OH, 1975; p 515.

(30) Zhong, B.; Yin, J.; Jia, Y.; Chen, L.; Hang, Y.; Yin, J. Laser Cooling of Yb ${ }^{3+}$-Doped LuLiF $_{4}$ Crystal. Opt. Lett. 2014, 39, 2747.

(31) Volpi, A.; Meng, J.; Albrecht, A. R.; Sheik-Bahae, M.; Biner, D.; Krämer, K. W.; Hehlen, M. P. Bridgman Growth of $\mathrm{LiYF}_{4}$ and $\mathrm{LiLuF}_{4}$ Crystals for Radiation-Balanced Lasers. Photonic Heat Engines Sci. Appl. 2019; p 24.

(32) Jia, Y. Crystal Radii and Effective Ionic Radii of the Rare Earth Ions. J. Solid State Chem. 1991, 95, 184-187.

(33) Bowman, S.; O’Connor, S.; Biswal, S. Radiation Balanced Lasers. CLEO/Europe. 2005 Conf. Lasers Electro-Optics Eur. 2005. pp 35-35.

(34) Ding, M.; Zhu, F.; Ma, D.; Huang, X.; Liu, P.; Song, K.; Zhong, J.; Xi, J.; Ji, Z.; Chen, D. KF-Mediated Controlled-Synthesis of Potassium Ytterbium Fluorides (Doped with $\mathrm{Er}^{3+}$ ) with Phase-Dependent Upconversion Luminescence. CrystEngComm 2015, 17, $7182-7190$.

(35) Hehlen, M. P. Crystal-Field Effects in Fluoride Crystals for Optical Refrigeration. 2010; p 761404.

(36) Karbowiak, M.; Gnutek, P.; Rudowicz, C. Energy Levels and Crystal Field Parameters for $\mathrm{Nd}^{3+}$ Ions in $\mathrm{BaY}_{2} \mathrm{~F}_{8}, \mathrm{LiKYF}_{5}$, and $\mathrm{K}_{2} \mathrm{YF}_{5}$ Single Crystals. Spectrochim. Acta Part A Mol. Biomol. Spectrosc. 46-60.

(37) Henderson, B.; Bartram, R. H. Crystal-Field Engineering of Solid-State Laser Materials; Cambridge University Press: Cambridge, 2000.

(38) Saha, S.; Chowdhury, P. S.; Patra, A. Luminescence of $\mathrm{Ce}^{3+}$ in $\mathrm{Y}_{2} \mathrm{SiO}_{5}$ Nanocrystals: Role of Crystal Structure and Crystal Size. J. Phys. Chem. B 2005, 109, 2699-2702. 
(39) Eichhorn, M. Fluorescence Reabsorption and Its Effects on the Local Effective Excitation Lifetime. Appl Phys B 2009, 96, 369-377.

(40) Xue, X.; Uechi, S.; Tiwari, R. N.; Duan, Z.; Liao, M.; Yoshimura, M.; Suzuki, T.; Ohishi, Y. Size-Dependent Upconversion Luminescence and Quenching Mechanism of $\mathrm{LiYF}_{4}: \mathrm{Er}^{3+} / \mathrm{Yb}^{3+}$ Nanocrystals with Oleate Ligand Adsorbed. Opt. Mater. Express 2013, 3, 989 .

(41) Sumida, D. S.; Fan, T. Y. Effect of Radiation Trapping on Fluorescence Lifetime and Emission Cross Section Measurements in Solid-State Laser Media. Opt. Lett. 1994, 19, 1343.

(42) Zhou, X.; Xia, X.; Smith, B. E.; Lim, M. B.; Bard, A. B.; Pant, A.; Pauzauskie, P. J. Interface-Dependent Radiative Lifetimes of $\mathrm{Yb}^{3+}, \mathrm{Er}^{3+} \mathrm{Co}-$ Doped Single $\mathrm{NaYF}_{4} \mathrm{Up}^{-}$ conversion Nanowires. ACS Appl. Mater. Interfaces 2019, 11, 22817-22823.

(43) Pant, A.; Smith, B. E.; Crane, M. J.; Zhou, X.; Lim, M. B.; Frazier, S. A.; Davis, E. J.; Pauzauskie, P. J. Optomechanical Thermometry of Nanoribbon Cantilevers. J. Phys. Chem. C 2018, 122, 7525-7532.

(44) Pant, A.; Xia, X.; Davis, E. J.; Pauzauskie, P. J. Solid-State Laser Refrigeration of a Semiconductor Optomechanical Resonator. arXiv Prepr. arXiv1910.02153 2019,

(45) Roder, P. B.; Smith, B. E.; Zhou, X.; Crane, M. J.; Pauzauskie, P. J. Laser Refrigeration of Hydrothermal Nanocrystals in Physiological Media. Proc. Natl. Acad. Sci. 2015, 112, 15024-15029. 


\section{Graphical TOC Entry}

Some journals require a graphical entry for the Table of Contents. This should be laid out "print ready" so that the sizing of the text is correct.

Inside the tocentry environment, the font used is Helvetica 8 pt, as required by Journal of the American Chemical Society.

The surrounding frame is $9 \mathrm{~cm}$ by $3.5 \mathrm{~cm}$, which is the maximum permitted for Journal of the American Chemical Society graphical table of content entries. The box will not resize if the content is too big: instead it will overflow the edge of the box.

This box and the associated title will always be printed on a separate page at the end of the document. 